\title{
La cultura empresarial y las incapacidades de aprendizaje organizacional en las Mipymes de Durango Capital
}

Business Culture and Incapacity of Organizational Learning in SMES of Durango Capital

\section{A cultura empresarial e as incapacidades de aprendizagem organizacional nas Mipymes de Durango Capital}

\section{José Gerardo Ignacio Gómez Romero*}

Fecha de recibido: 21 de julio de 2016

Fecha de aprobado: 16 de diciembre de 2016

Doi: http://dx.doi.org/10.12804/revistas.urosario.edu.co/empresa/a.4969

Para citar: Gómez Romero, J. G. (2017). La cultura empresarial y las incapacidades de aprendizaje organizacional en las Mipymes de Durango Capital. Universidad \& Empresa, 19(33), $113-136$. Doi: http://dx.doi. org/10.12804/revistas.urosario.edu.co/empresa/a.4969

\section{RESUMEN}

El objetivo del presente trabajo se centra en determinar la posible relación entre la cultura empresarial y las incapacidades de aprendizaje organizacional en la micro, pequeña y mediana empresa (Mipymes) de la ciudad de Durango, así como establecer cuál es la cultura empresarial dominante y la incapacidad de aprendizaje organizacional que con mayor frecuencia se presenta en las empresas estudiadas.

Para lograr lo anterior, se aplicó un cuestionario basado en Yeung, Ulrich, Nason y Von Glinow (2000), adaptado y validado a un entorno mexicano por Gómez-Romero (2008), a una muestra de 200 Mipymes, diagnosticándose ambas variables aplicando análisis de la varianza (Anova), y para determinar la relación se utilizaron modelos de regresión lineal.

\footnotetext{
Doctor en Administración, Universidad Juárez del Estado de Durango (México). Correo electrónico: gerardoignaciog@yahoo.com.mx
} 
Realizados los análisis correspondientes, se encontró evidencia a favor de la relación significativa de la cultura y las incapacidades de aprendizaje organizacional.

Palabras clave: cultura empresarial, incapacidades de aprendizaje, Mipymes.

\section{ABSTRACT}

The objective of this paper focuses on determine the possible relationship between business culture and learning organizational incapacities in micro, small and medium enterprises (SMEs) in the city of Durango, as well as stablish which is the dominant business culture and the organizational learning incapacity that is presented with more frequency in the studied enterprises.

To achieve this a questionnaire based in Yeung, Ulrich, Nason and Von Glinow (2000), adapted and validated in the Mexican context by Gómez Romero (2008), was applied to a sample of 200 SMEs diagnosing both variables and applying analysis of variance (Anova) and to determine the relationship lineal regression models were used. Once the appropriate analyses were done, it was found evidence in favor of the significate relationship between business culture and learning organizational incapacities.

Keywords: business culture, learning incapacities, SMES.

\section{RESUMO}

O objetivo do presente trabalho centra-se em determinar a possível relação entre a cultura empresarial e as incapacidades de aprendizagem organizacional na micro, pequena e mediana empresa (Mipymes) da cidade de Durango, assim como estabelecer qual é a cultura empresarial dominante e a incapacidade de aprendizagem organizacional que com maior frequência se apresenta, nas empresas estudadas.

Para conseguir o anterior aplicou-se um questionário baseado em Yeung, Ulrich, Nason e Von Glinow (2000), adaptado e validado a um entorno mexicano por Gómez (2008), a uma amostra de 200 Mipymes, diagnosticando-se ambas as variáveis aplicando análise da variância (Anova), e para determinar a relação utilizaram-se modelos de regressão lineal. Realizadas as análises correspondentes, encontrou-se evidência a favor da relação significativa da cultura e as incapacidades de aprendizagem organizacional.

Palavras-chave: cultura empresarial, incapacidades de aprendizagem, Mipymes. 


\section{INTRODUCCIÓN}

El objetivo del presente trabajo se centra en determinar la posible relación entre la cultura empresarial y las incapacidades de aprendizaje organizacional en la micro, pequeña y mediana empresa (Mipymes) de la ciudad de Durango, así como establecer cuál es la cultura empresarial dominante y la incapacidad de aprendizaje organizacional que con mayor frecuencia se presenta en las empresas estudiadas.

El objeto de estudio es la Mipyme; la importancia de estudiar este tipo de empresas se desprende de la información que presenta el Directorio Estadístico Nacional de Unidades Económicas (DENUE) del Instituto Nacional de Estadística e Informática (INEGI) (2014b), que muestra que en México existen más de tres millones de empresas, de las cuales el 95,7\% son microempresas, el 3,1\% son pequeñas empresas y el $0,9 \%$ son medianas, que sumadas dan un total del $99,7 \%$ de Mipymes, y que generan el $64 \%$ del empleo del país y producen el $42 \%$ de la riqueza del producto interno bruto (PIB).

En ese sentido, autores como Furtado (2003), Dallago (2003), Gooderham (2007), Audretsch y Mahmood (1995), destacan su importancia en aspectos como la generación de em- pleos, su aporte a la economía y el impacto social que tienen, además de señalar algunos problemas que las aquejan, como la informalidad, una deficiente administración y una mortandad acelerada, por lo que su relevancia está fuera de discusión.

\section{PLANTEAMIENTO DEL PROBLEMA}

Cayeux (1964), Huntington (2001) y Palomo (2011) coinciden al afirmar que en la actualidad vivimos una nueva época, denominada del conocimiento y la información, donde el aprendizaje juega un factor importante como fuente de poder, de modo que quien logre generarlo de la mejor manera tendrá más posibilidades de triunfar en una sociedad globalizada y altamente competitiva.

El aprendizaje de las organizaciones se genera cuando sus miembros reaccionan y dan respuesta a los cambios que se verifican en el medio ambiente; cuando detectan necesidades, errores y los corrigen; pero cuando no son capaces de reaccionar ante los cambios y exigencias de un entorno volátil y cambiante, hipotecan su futuro y el de las organizaciones, de manera tal que una de las causales de ello es producto de las incapacidades o barreras de aprendizaje organizacional. 


\subsection{Objetivo, pregunta de investigación e hipótesis}

Objetivo: determinar la posible relación entre la cultura empresarial y las incapacidades de aprendizaje organizacional en las Mipymes de la ciudad de Durango, así como establecer cuál es la cultura empresarial dominante y la incapacidad de aprendizaje organizacional que con mayor frecuencia se presenta en las empresas estudiadas.

- Preguntas de investigación

¿Existe una relación entre la cultura empresarial y las incapacidades de aprendizaje organizacional en las Mipymes de Durango Capital?

¿Cuál es la cultura empresarial dominante en las Mipymes de Durango Capital?

En caso de presentarse, ¿qué incapacidades se presentan en mayor medida y más significativamente en las Mipymes de Durango Capital?

\section{- Hipótesis}

H1: Existe una relación significativa entre la cultura empresarial y las incapacidades de aprendizaje organizacional en las Mipymes de Durango Capital.

H2: La cultura adhocrática es la dominante en las Mipymes de Durango Capital.

H3: La ceguera es la incapacidad que con más frecuencia se presenta en las Mipymes de Durango Capital.

- Justificación

La tabla 1, elaborada con datos del INEGI (2006), muestra la población ocupada por las Mipymes en Durango en comparación con la empleada por las grandes empresas, donde destaca la capacidad de las micro, pequeñas y medianas empresas en la generación de empleos en Durango.

Con relación a lo anterior, Prater y Ghosh (2005) referencian un informe de la Organización de las Naciones Unidas (ONU) donde se afirma que la micro, pequeña y mediana empresa en los países en desarrollo genera más del $90 \%$ de los empleos, y en los países desarrollados más del $50 \%$. Particularmente en Durango generan más del $75 \%$ de los empleos, de manera tal que son de vital importancia en la actividad económica. 
Tabla 1. Personal ocupado por tipo de empresa en Durango

\begin{tabular}{|c|c|c|}
\hline Tipo de empresa o de sector & \multicolumn{2}{|c|}{$\%$ de población que emplea } \\
\hline Microempresa & \multirow{3}{*}{$\begin{array}{l}46,90 \\
18,30 \\
10,40\end{array}$} & \multirow{3}{*}{75,60} \\
\hline Pequeña empresa & & \\
\hline Mediana empresa & & \\
\hline Grande empresa & \multicolumn{2}{|c|}{12,20} \\
\hline Gobierno & \multicolumn{2}{|c|}{7,70} \\
\hline Sector agrícola & \multicolumn{2}{|c|}{4,50} \\
\hline Total de personal ocupado & \multicolumn{2}{|c|}{100} \\
\hline
\end{tabular}

Fuente: INEGI. (2015). Perspectiva económica Durango.

Con datos del Sistema de Información Empresarial Mexicano (SIEM, 2015) del INEGI, se elabora la tabla 2 , donde se puede observar el número de unidades empresariales registradas en Durango y donde destaca el número de Mipymes.

Tabla 2. Número de unidades empresariales en Durango

\begin{tabular}{|c|c|c|c|c|}
\hline & Industrial & Comercial & Servicio & Total \\
\hline Micro & 38044 & 444798 & 162367 & 645209 \\
\hline Pequeña & 7941 & 11310 & 14501 & 33752 \\
\hline Mediana & 2902 & 2846 & 1752 & 7500 \\
\hline Grande & 1252 & 941 & 1553 & 3746 \\
\hline Total & 50139 & 459895 & 180173 & 690207 \\
\hline
\end{tabular}

Fuente: SIEM (2015) del INEGI.

Lo anterior destaca la importancia de las Mipymes, lo que contrasta con el tratamiento y los apoyos que este tipo de empresas reciben de parte del gobierno, así como de las instituciones financieras, pues en la medida en que estas empresas son más pequeñas, más desprotegidas se encuentran y, como consecuencia, es más fácil que desaparezcan de los mercados de trabajo.
Todo esto se convierte en un círculo vicioso, que influye de manera desmedida en su mortandad, al presentarse la suma de los siguientes factores: la carencia o limitada capacidad técnica de los dueños o encargados más un escaso o magro financiamiento, más miedo a explorar nuevas cosas, más una competencia cada vez más voraz, más un entorno dinámico y cambiante, que dan como resultado 
una incapacidad para crecer o para afrontar a la competencia, que generalmente termina en el cierre del negocio.

Derivado de lo anterior, es necesario trabajar con estas empresas para auxiliarlas, y generar una cultura de información y de mutua colaboración que les permita sobrevivir, y de ser necesario que aprendan a producir conocimiento organizacional y a descubrir sus incapacidades de aprendizaje organizacional.

\section{REVISIÓN DE LITERATURA}

El aprendizaje es una facultad eminentemente humana, el hombre aprende cuando aprehende, es decir, cuando atrapa información del medio ambiente, se lo apropia y lo enriquece con su experiencia personal, y termina traduciéndolo en diversas formas de resolver problemas, así como en nuevas maneras de realizar cosas; a este nuevo conocimiento se llama adquisición de competencias individuales (Alles, 2001), que no es otra cosa que un conocimiento nuevo o mejorado.

Cuando ese conocimiento se utiliza para resolver problemas de mejor manera, solo es conocimiento individual, pero cuando es compartido a lo largo y ancho de la organización con otros miembros, se convierte en conocimiento organizacional. Al respecto, Choo (1999) afirma que cuando el aprendizaje se traslada más allá del aprender individual, e implica además la capacidad para asimilar las ideas nuevas de otros, entonces se da el aprendizaje organizacional.

De ello podemos concluir que el conocimiento reside en la mente de los seres humanos y solo ellos pueden crearlo, que las organizaciones y los negocios generan conocimiento merced a que los seres humanos se insertan e interactúan en ellos, se convierten en su parte esencial y que, al socializarlo en la organización, se convierte en conocimiento organi-zacional. Alcover (2002) describe tres tipos de aprendizaje, a los que llama: aprendizaje correctivo (de bucle sencillo); aprendizaje adaptativo (de doble bucle) y aprendizaje como solución de problemas (de triple bucle). Estos tres tipos de aprendizaje representan la evolución del conocimiento individual que se transforma en conocimiento grupal.

Enlaactualidad, las empresas enfrentan un entorno impredecible, cambiante e incierto, caracterizado por la complejidad y el dinamismo, y no les queda otra opción que interactuar conél, debiendo adaptarse al ambiente en forma continua si quieren sobrevivir (Daft, 2000). En el entorno 
se encuentra el objetivo primordial de toda organización, los clientes o usuarios, y si la respuesta que la empresa da a sus clientes es errónea, será el inicio de su desaparición de los mercados, como lo señalan Choo (1999) y Gómez, Villarreal y Villarreal (2014).

Probst, Raub y Romhardt (2001) afirman que, para sobrevivir y competir en la sociedad del conocimiento, las empresas deben aprender a manejar los activos intelectuales con los que cuentan, por el papel relevante que juegan en el incremento de la productividad, la innovación y la generación de conocimiento en el trabajo, y esta aportación generalmente se da en la creación y difusión del know-how, como lo sostienen Bounfour y Edvinsson (2005).

Bustillo (2003) y Argote (2013) señalan que el aprendizaje es siempre una actividad voluntaria que busca conseguir una mejora y nunca debe ser impuesta, porque si eso ocurre los resultados quedan hipotecados. Teniendo en cuenta lo anterior, es esencial resaltar la actitud que guarden los directivos ante este tema, pues son ellos la clave para que la generación de conocimiento se dé o se estanque, puesto que "la prueba última de la capacidad para aprender, es la medida en la que los directivos demuestran, por medio de sus actos, un compromiso con la generación y la generalización de ideas" (Yeung et al., 2000, p. 160).

Gómez-Romero (2008) sostiene que losadministradores que quieran crear organizaciones que aprendan deben concentrarse en el aprendizaje, tanto por parte de los individuos como de la organización. Sin embargo, se debe considerar que el aprendizaje ocurre cuando se identifica correctamente la cultura de la organización, y a partir de ahí, se asegura que esté en línea con la estrategia general del negocio y su contexto. Argyris y Schön (1998) indican que el aprendizaje organizacional les permite a las organizaciones adaptarse a los cambios y evitar repetición de errores pasados.

Yeung et al. (2000) argumentan que no es casualidad que la mayoría de las organizaciones aprendan mal, y coinciden con Argyris (1991) y Pérez y Cortés (2007) al afirmar que la incipiente formación administrativa de los gerentes y dueños de los negocios acarrea necesariamente deficiencias en la generación del conocimiento organizacional (Co). A estas deficiencias en la generación de CO, se les llama barreras o incapacidades de aprendizaje.

Las incapacidades impiden que el aprendizaje sea efectivo, son como 
una enfermedad silenciosa, que evita que muchos procesos se realicen de manera correcta y asertiva, no provoca aparentemente ningún síntoma, pero cuando ha invadido totalmente a la organización, termina por hacerla desaparecer. Además, frenan la capacidad de las organizaciones, pues les impiden rehacerse por medio de la realimentación, y de aprender de sus errores, y, como consecuencia, no dan respuesta adecuada a las demandas de los clientes.

Las incapacidades o barreras de aprendizaje, como se les conoce, se refieren a condiciones que dificultan u obstaculizan el aprendizaje en el ámbito de las organizaciones y de los negocios. Yeung et al. (2000) afirman que el aprendizaje organizacional tiene dos etapas o momentos:

1) la generación del conocimiento y 2) la generalización, y la suma de estas etapas busca transformar a la organización en una organización que aprende.

De la revisión de la literatura se encontró que los autores que estudian el tema de barreras coinciden en los efectos que estas provocan, aunque las llamen de diferentes nombres. Haremos un breve recorrido de los principales autores de este tema y al final presentaremos las que distinguen Yeung et al. (2000), que serán uno de los modelos en el que se basará el presente estudio.
Senge (1993) describe siete barreras, a las que llama:

- "Yo soy mi puesto", se presenta cuando las personas solo se preocupan de manera aislada por las tareas de su puesto y no las vinculan con el resto de las actividades de la organización.

- "El enemigo externo", se da como resultado de culpar de los errores a los demás y no asumir la responsabilidad de los propios actos.

- "La ilusión de hacerse cargo", es solucionar de manera simulada los problemas de la empresa.

- "La fijación en los hechos", condena a la organización a resolver problemas de manera reactiva.

- "La parábola de la rana hervida", es la dificultad de ver los cambios graduales y constantes del entorno.

- "La ilusión de que se aprende con la experiencia", es el resultado de pensar que se aprende mejor de la experiencia propia, poniendo en riesgo la integridad de la organización.

- "El mito del equipo administrativo", parte del pensamiento erróneo de que el equipo directivo está formado por personas capacitadas para resolver todos los problemas.

Por su parte, March y Leavitt (1988, citados por Argyris, 1999) identifican dos barreras que dificul- 
tan el aprendizaje: el "aprendizaje supersticioso", que sostiene que determinadas rutinas o formas de hacer conducen al logro de resultados exitosos, sin tener ninguna prueba que evidencie esto; las "trampas de competencia", que son el resultado de pensar que la habilidad adquirida, aunque esté mal, convertirá a las personas en expertos.

Geranmayeh (1992) distingue dos barreras a las que llama: el "mito de la infalibilidad", fundado en la infalibilidad de los directivos; y "matar al mensajero", inhibiendo con esto la participación de señalar errores y de mejorar.

Argyris (1993) describe cinco barreras, que son: la "incompetencia competente", que busca resolver problemas nuevos con soluciones viejas; las "rutinas defensivas", son la negación de reconocer los errores que se cometen y sus consecuencias; el "autoengaño", que es la ceguera para ver lo que está mal; y el "malestar organizativo", que es consecuencia del autoengaño, al no aceptar las incongruencias y los errores, creando un clima de malestar generalizado.

Probst y Büchel (1995) coinciden con el planteamiento de Argyris (1993), y agregan dos incapacidades más: "privilegios y tabúes", resultante de algunos privilegios que obtienen algunos miembros que bloquean el aprendizaje al resistirse a renunciar a esas prerrogativas; y la "patología de la información", cuando no se sabe manejar adecuadamente la información con la que se cuenta para la buena toma de decisiones.

Lounamaa y March (1987) plantean de manera genérica "el dilema del aprendizaje", que consiste en pensar que la resolución de problemas de corto plazo resolverá el rumbo a largo plazo, sin pensar en el futuro.

Watkins y Marsick (1993) señalan tres barreras: "la incapacidad aprendida", resultado de la pasividad y desmotivación ante las oportunidades de aprendizaje; la "visión de túnel", cuando pensamos que somos únicos en el mercado y no tenemos competencia; y el "aprendizaje truncado", como resultado de la resolución - estética- de problemas, sin atender las causas de fondo.

Por último, la propuesta que hacen Yeung et al. (2000), quienes plantean siete incapacidades, las cuatro primeras impiden la generación del conocimiento y las tres restantes se oponen a su generalización:

- Ceguera: impide evaluar correctamente las amenazas y oportunidades del entorno. 
- Candidez: exhibe deficiencias en el análisis de las situaciones, pues ante situaciones complejas se aplican las reglas de cajón, sin un análisis cuidadoso.

- Homogeneidad: solo toma en cuenta el punto de vista del dueño o gerente, quien es omnisciente y experto en todo.

- Acoplamiento estrecho: la inflexibilidad y la no adaptabilidad es la tónica, pues se deriva de análisis de la realidad cándidos e incompletos.

- Parálisis: impide implantar soluciones, porque se emplea demasiado tiempo en su análisis.

- Aprendizaje de supersticiones: resulta de culpar a terceras personas de lo malo que ocurre en el negocio.

- Difusión deficiente: es el reflejo de una deficiente comunicación organizacional, dejando que el rumor y la incertidumbre rijan.

De la literatura revisada, se puede concluir que las incapacidades de aprendizaje son afectadas en mayor o menor medida por una serie de factores endógenos y exógenos de la organización; los estudiosos le han dado el nombre de cultura, que para efectos del presente estudio se considera la cultura propia de las organizaciones empresariales.

Hernández-Romo (2004, p. 14) la define como "la acumulación de significados socialmente entendidos y que diferentes actores como los empresarios, emplean para configurar significados en la actividad diaria, movilizando estos códigos, recreando significados y legitimando valores colectivos dando así sentido al mundo de la empresa, la economía, la familia y la sociedad; se trata de la comprensión del significado subjetivo de la conducta humana".

Para Deal y Kennedy (2000), la cultura empresarial o corporativa está compuesta por el medio ambiente empresarial, el conjunto de valores y creencias, héroes, personajes que encarnan dichos valores, ritos y rituales, así como la red cultural integrada por las comunicaciones informales, a través de las cuales se relacionan los mencionados elementos.

Thevenet (1992) afirma que la cultura es tan importante que ningún estudio que aborde la problemática empresarial estará completo si no considera a la cultura empresarial como parte fundamental, ya que, según plantea Fernández (1995, citado en Hernández, Mendoza y González, 2008), explica lo que otros recursos no pueden acerca de la situación de la empresa. Lo anterior tiene sintonía con Bohmer y Edmondson (2001) y con Díaz-Nieto (2008), cuando sostienen que tiene una relación directa con el aprendizaje organizacional, puesto que la 
habilidad para aprender es esencial en un entorno cambiante e incierto y la cultura de la organización puede facilitar o retardar el aprendizaje colectivo.

Debemos recordar que la cultura es intangible, sin embargo, se puede percibir a través de sus expresiones, es decir, los artefactos visibles que son consecuencia de esta y también ayudan a interpretarla, por medio de inferencias con base en estas manifestaciones (Daft, 1998). Estas manifestaciones también se aprecian en sus ritos y ceremonias, que representan a la cultura en acción, como lo comenta Deal y Kennedy (2000) y Armenteros, Guerrero, Noyola y Molina (2012).

Uno de los problemas que enfrenta la cultura es el descrito por Bower (1995, citado en Pirela y Sánchez, 2009), cuando declara que esta es un elemento invisible para las personas que pertenecen a las organizaciones, y que, sin embargo, de acuerdo con Pfeffer (2000), es un cuerpo o tradición aprendida, que rige lo que se necesita saber, pensar y sentir para cumplir con las normas a fin de convertirse en miembro del grupo. Considerando estas características, resulta fácil comprender que no siempre estos son conscientes de su presencia y de su importancia, puesto que, conforme con Rodríguez y Ramírez (2004), influye en aspectos tales como las conductas laborales.

Así como los individuos tienen personalidad, algunos autores consideran que también las organizaciones la tienen (Bridges, 2000; Fernández y Hogan, 2003, citados en Calderón y Naranjo, 2007). Por su lado, Guzzeta (2008) asevera que, cuanto más incierto sea el entorno en que se desenvuelve la empresa, más flexible y dinámica deberá ser la organización interna, solo así será capaz de adecuarse al escenario de rápidos cambios en que actúa.

Quinn, Anderson y Finkelstein (1998), Quinny McGrath(1985), así como Cameron y Freeman (1991), citados en Cameron y Quinn (2006), coinciden cuando afirman que, debido a que las culturas están definidas por valores, suposiciones e interpretaciones de los miembros de la organización, es posible que se pueda obtener un modelo de tipos de cultura - criterio empleado en este estudio-, que en este caso se denomina 'modelo de valores rivales', el cual ha sido de gran importancia en el avance del estudio de los aspectos culturales, a través de las tipologías que se han identificado y a las que se ha denominado como clan, jerarquía, mercado $\mathrm{y}$ adhocracia. 
En cuanto a sus características, la cultura de jerarquía se centra en los aspectos internos de la empresa, por lo que aprecia el control y la estabilidad, sobre la flexibilidad; la cultura de mercado se enfoca hacia el exterior y los resultados, por lo que sus participantes son competitivos y orientados a los objetivos, antes que hacia los asuntos internos, sin embargo, valoran la estabilidad y el control; en la cultura de clan, sus participantes se enfocan en sus asuntos internos, valorando la flexibilidad, y sus líderes son considerados como mentores y aun como figuras paternas; en cuanto a la cultura de adhocracia, esta se encuentra en aquellas empresas que se centran en aspectos externos, pero valora un alto grado de flexibilidad, innovación y experimentación, antes que la estabilidad y el control.

Estas descripciones corresponden a tipos ideales de cultura, sin embargo, existe evidencia empírica que muestra la dificultad de encontrar tipos de cultura únicos en las organizaciones, existiendo la posibilidad de una cultura dominante coexistiendo con otros tipos de cultura.

Se destaca que este modelo fue elegido porque, de acuerdo con Hernández, Méndez y Contreras (2014), es un encuadre teórico que aglutina los principales enfoques administra- tivos, además de contar con abundante evidencia empírica. A partir de estos conceptos, se han podido construir instrumentos con qué medir estos arquetipos culturales, lo que a su vez ha abierto el camino a estudios empíricos que puedan profundizar en aspectos tales como cuál o cuáles son los tipos de cultura predominantes que caracterizan a las empresas de éxito.

\section{METODOLOGÍA}

El presente estudio es correlacional, porque trata de establecer la relación entre variables, según Hernández, Fernández y Baptista (2006); también es transversal, porque la toma de información se limitó a una sola ocasión; y es no experimental, porque se obtiene y analiza la información sin modificar ninguna de las condiciones existentes.

El cuestionario que se aplicó en la presente investigación lo estructuraron Yeung et al. (2000), y por ser un cuestionario probado y validado en su estudio en 300 empresas alrededor del mundo, también se validó en el contexto mexicano y fue aplicado por Gómez-Romero (2008). Consta de 50 reactivos (34 referentes a las siete incapacidades de aprendizaje y 16 a los cuatro tipos de cultura), con una escala tipo Likert de seis opciones, gradualmente ascendiendo de 
la siguiente forma: "No sé/no aplica, muy poco, poco, algo, mucho y muchísimo".

Elobjetodel presenteestudioeslaMipyme. Para determinar el universo, se conjuntaron varios directorios de empresas de instituciones federales, como el Instituto de Fomento Nacional para la Vivienda de los Trabajadores (Infonavit), la Secretaría de Economía en su delegación Durango (SE), la Secretaría del Trabajo y Previsión Social delegación Durango (STPS); de organismos empresariales, como la Cámara Nacional de Comercio (Canaco) y la Confederación de Patrones de la República Mexicana (Coparmex). Esto se hizo ya que en la localidad ninguna institución pública o privada cuenta con un padrón completo y confiable.

Una vez estructurado el directorio, que ascendió a 1400 empresas con asentamiento en la capital del Estado, para determinar la muestra de estudio se calculó aplicando el programa Stats V. 9, dando como resultante 325 empresas por estudiar, mismas que fueron escogidas aleatoriamente.

La muestra integró mandos medios en adelante (dueños, gerentes, subgerentes, jefes de departamentos y encargados) de 179 microempresas, 98 pequeñas y 48 medianas de los tres sectores, industrial, comercial y de servicios.

Se determinó la prueba piloto en 30 empresas y se les aplicó el cuestionario, se obtuvo realimentación para ver si no había algún tipo de dificultad para entender el cuestionario, lo que nos permitió hacer algunos ajustes en la redacción de las preguntas; luego procedimos a la recolección de la información.

Se visitó cada uno de los negocios, entablando una charla con los dueños o encargados, entregándoles una carta de presentación institucional, donde se les explicó y se pidió su colaboración en el proyecto, además de plantearles brevemente los objetivos del estudio de forma oral, aclarando los fines académicos, y el entrevistador coadyuvó en el llenado del cuestionario.

Obtenidos los datos, se trabajaron en el programa estadístico sPss versión 19.0, creando una base de datos y calculando la confiabilidad del instrumento por medio de la prueba alfa de Cronbach, cuyo resultado se puede apreciar en la tabla 3. El valor del alfa de Cronbach por debajo de 0,60 es mediocre, de 0,60 a 0,65 es indeseable, de 0,65 a 0,70 es aceptable, de 0,70 a 0,80 es respetable y de 0,80 a 0,90 es muy buena, de acuerdo con Barraza-Macías (2008). Es 
necesario destacar que esta prueba los y cultura), siendo cada una intese aplicó para cada dimensión (esti- grada por varias variables.

Tabla 3. Confiabilidad del instrumento de medición

\begin{tabular}{|l|c|}
\hline \multicolumn{1}{|c|}{ Constructo } & Valor de alfa \\
\hline Incapacidades de aprendizaje & 0,848 \\
\hline Cultura & 0,888 \\
\hline
\end{tabular}

Fuente: elaboración del autor.

\section{RESULTADOS}

Para obtener el diagnóstico de la cultura y de las incapacidades dominantes, se obtuvieron los prome- dios para cada indicador, lo cual nos muestra una descripción del tipo de cultura, así como de las incapacidades. Estos resultados se muestran en las tablas 4 y 5 .

Tabla 4. Promedios para cada tipo de cultura

\begin{tabular}{|c|c|c|c|c|}
\hline Tipos de cultura & Clan & Adhocracia & Mercado & Jerárquica \\
\hline Promedios & 4,0 & 4,75 & 4,5 & 2,75 \\
\hline
\end{tabular}

Fuente: elaboración del autor.

Tabla 5. Promedios para cada tipo de incapacidad de aprendizaje

\begin{tabular}{|c|c|c|c|c|c|c|c|}
\hline 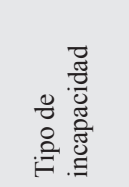 & 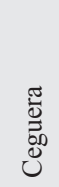 & 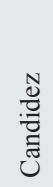 & 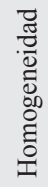 & 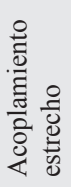 & 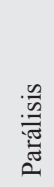 & 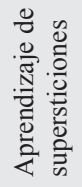 & 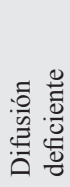 \\
\hline Promedio & 3,6 & 3,5 & 3,1 & 3,0 & 3,2 & 3,0 & 3,5 \\
\hline
\end{tabular}

Fuente: elaboración del autor.

También se obtuvo la frecuencia estos resultados se muestran en las tanto del tipo de cultura como del tablas 6 y 7 . tipo de incapacidad de aprendizaje; 
Tabla 6. Frecuencias de acuerdo con el tipo de cultura empresarial

\begin{tabular}{|l|c|}
\hline \multicolumn{1}{|c|}{ Tipos de cultura } & Frecuencia \\
\hline Clan & 55 \\
\hline Adhocrática & 114 \\
\hline Jerárquica & 52 \\
\hline Mercado & 104 \\
\hline Total & 325 \\
\hline
\end{tabular}

Fuente: elaboración del autor.

Tabla 7. Frecuencias de acuerdo con el tipo de incapacidad de aprendizaje

\begin{tabular}{|l|c|c|}
\hline \multicolumn{1}{|c|}{ Tipo de incapacidad } & Frecuencia & Promedios \\
\hline Ceguera & 159 & 3,6 \\
\hline Candidez & 42 & 3,5 \\
\hline Homogeneidad & 10 & 3,1 \\
\hline Acoplamiento estrecho & 26 & 3 \\
\hline Parálisis & 13 & 3,2 \\
\hline Aprendizaje de supersticiones & 20 & 3 \\
\hline Difusión deficiente & 55 & 3,5 \\
\hline
\end{tabular}

Fuente: elaboración del autor.

Posteriormente, para saber si existen diferencias estadísticamente significativas entre los grupos correspondientes a cada tipo de cultura, se aplicó un análisis de la varianza (Anova)con una prueba $F$, que, según Lind, Marchal y Wathen (2005), se utiliza para probar si dos muestras provienen de poblaciones que tienen varianzas iguales (la cual se considera hipótesis nula). Los resultados de estas pruebas se observan en las tablas 8 y 9.

Tabla 8. Anova de tipos de cultura empresarial

\begin{tabular}{|l|c|c|c|c|c|c|}
\hline \multicolumn{2}{|c|}{} & $\begin{array}{c}\text { Suma de } \\
\text { cuadrados }\end{array}$ & Gl & $\begin{array}{c}\text { Media } \\
\text { cuadrática }\end{array}$ & F & Sig. \\
\hline \multirow{2}{*}{$\begin{array}{l}\text { Cultura de } \\
\text { mercado } \\
\text { promedio }\end{array}$} & Intergrupos & 92036 & 22 & 4183 & 85664 & 0,000 \\
\cline { 2 - 7 } & intragrupos & 8644 & 302 & 0,049 & & \\
\cline { 2 - 7 } & Total & 100680 & 324 & & & \\
\hline
\end{tabular}




\begin{tabular}{|l|c|c|c|c|c|c|}
\hline \multicolumn{2}{|c|}{} & $\begin{array}{c}\text { Suma de } \\
\text { cuadrados }\end{array}$ & Gl & $\begin{array}{c}\text { Media } \\
\text { cuadrática }\end{array}$ & F & Sig. \\
\hline \multirow{2}{*}{$\begin{array}{l}\text { Cultura } \\
\text { adhocrática } \\
\text { promedio }\end{array}$} & Intergrupos & 79418 & 22 & 3610 & 17951 & 0,000 \\
\cline { 2 - 7 } & Intragrupos & 35595 & 302 & 0,201 & & \\
\cline { 2 - 8 } & Total & 115014 & 324 & & & \\
\hline \multirow{3}{*}{$\begin{array}{l}\text { Cultura de clan } \\
\text { promedio }\end{array}$} & Intergrupos & 58363 & 22 & 2653 & 11785 & 0,000 \\
\cline { 2 - 8 } & Intragrupos & 39842 & 302 & 0,225 & & \\
\cline { 2 - 8 } & Total & 98205 & 324 & & & \\
\hline \multirow{2}{*}{$\begin{array}{l}\text { Cultura } \\
\text { jerárquica } \\
\text { promedio }\end{array}$} & Intergrupos & 63024 & 22 & 2865 & 8594 & 0,000 \\
\cline { 2 - 8 } & Intragrupos & 59001 & 302 & 0,333 & & \\
\cline { 2 - 8 } & Total & 122025 & 324 & & & \\
\hline
\end{tabular}

Fuente: elaboración del autor.

Tabla 9. Anova de incapacidades de aprendizaje

\begin{tabular}{|l|c|c|c|c|c|c|}
\hline \multicolumn{2}{|c}{} & $\begin{array}{c}\text { Suma de } \\
\text { cuadrados }\end{array}$ & Gl & $\begin{array}{c}\text { Media } \\
\text { cuadrática }\end{array}$ & F & Sig. \\
\hline \multirow{4}{*}{ Experimentadoras } & Entre grupos & 68817 & 253 & 0,538 & 6225 & 0,000 \\
\cline { 2 - 8 } & Intragrupos & 6132 & 71 & 0,086 & & \\
\cline { 2 - 8 } & Total & 74950 & 324 & & & \\
\hline \multirow{3}{*}{$\begin{array}{l}\text { Adquisición de } \\
\text { competencias }\end{array}$} & Entre grupos & 128656 & 253 & 1005 & 6582 & 0,000 \\
\cline { 2 - 8 } & Intragrupos & 10842 & 71 & 0,153 & & \\
\cline { 2 - 8 } & Total & 139498 & 324 & & & \\
\hline \multirow{3}{*}{\begin{tabular}{l} 
Mejora continua \\
\cline { 2 - 8 }
\end{tabular}} & Entre grupos & 73897 & 253 & 0,577 & 5118 & 0,000 \\
\cline { 2 - 8 } & Intragrupos & 8009 & 71 & 0,113 & & \\
\cline { 2 - 8 } referencia & Total & 81905 & 324 & & & \\
\hline
\end{tabular}

Fuente: elaboración del autor.

Con el propósito de someter a prueba drados ordinarios para evaluar la las hipótesis de estudio, se aplicaron relación lineal de dependencia, que, modelos de regresión lineal usando de acuerdo con Hair, Anderson, el procedimiento de mínimos cua- Tatham y Black (2005), es una técnica 
de dependencia muy versátil y utilizada, aplicable en cualquier ámbito de los negocios, pudiendo ser empleada para analizar la rela- ción entre una variable criterio y una o varias variables independientes 0 predictoras; los resultados de estos cálculos se observan en la tabla 10.

Tabla 10. Resumen de los modelos de regresión

\begin{tabular}{|l|c|c|c|c|c|c|c|c|c|c|c|c|}
\hline $\begin{array}{c}\text { Var. } \\
\text { independiente }\end{array}$ & \multicolumn{3}{|c|}{ Clan } & \multicolumn{3}{c|}{ Adhocrática } & \multicolumn{3}{c|}{ Mercado } & \multicolumn{3}{c|}{ Jerárquica } \\
\hline 1 & 2 & 3 & 4 & 5 & 6 & 7 & 8 & 9 & 10 & 11 & 12 & 13 \\
\hline $\begin{array}{c}\text { Var. } \\
\text { dependiente }\end{array}$ & $\mathrm{r}^{2}$ & $\mathrm{~B}$ & Sig. & $\mathrm{r}^{2}$ & $\mathrm{~B}$ & Sig. & $\mathrm{r}^{2}$ & $\mathrm{~B}$ & Sig. & $\mathrm{r}^{2}$ & B & Sig. \\
\hline Ceguera & 0,154 & 0,455 & 0,000 & 0,012 & 0,118 & 0,122 & 0,097 & 0,357 & 0,000 & 0,492 & 0,731 & 0,000 \\
\hline Candidez & 0,089 & 0,267 & 0,000 & 0,164 & 0,335 & 0,000 & 0,065 & 0,225 & 0,000 & 0,020 & 0,114 & 0,045 \\
\hline Homogeneidad & 0,1 & 0,354 & 0,000 & 0,235 & 0,503 & 0,000 & 0,046 & 0,239 & 0,002 & 0,003 & 0,053 & 0,456 \\
\hline $\begin{array}{l}\text { Acoplamiento } \\
\text { estrecho }\end{array}$ & 0,07 & 0,318 & 0,000 & 0,208 & 0,508 & 0,000 & 0,035 & 0,222 & 0,008 & 0,005 & $-0,076$ & 0,324 \\
\hline Parálisis & 0,059 & 0,230 & 0,000 & 0,068 & 0,230 & 0,000 & 0,035 & 0,175 & 0,008 & 0,053 & 0,196 & 0,001 \\
\hline $\begin{array}{l}\text { Aprendizaje de } \\
\text { supersticiones }\end{array}$ & 0,031 & 0,213 & 0,013 & 0,068 & 0,292 & 0,000 & 0,005 & 0,084 & 0,324 & 0,020 & 0,155 & 0,045 \\
\hline $\begin{array}{l}\text { Difusión } \\
\text { deficiente }\end{array}$ & 0,297 & 0,612 & 0,000 & 0,276 & 0,545 & 0,000 & 0,159 & 0,442 & 0,000 & 0,046 & 0,215 & 0,002 \\
\hline
\end{tabular}

Fuente: elaboración del autor.

Cabe destacar que los modelos de regresión se aplicaron de la siguiente manera: se contrastaron cada tipo de cultura con cada tipo de incapacidad de aprendizaje, las variables independientes fueron los tipos de cultura. Lo anterior generó un conjunto de 28 modelos (cuatro tipos de cultura por siete incapacidades de aprendizaje).

\section{CONCLUSIONES}

En relación con las hipótesis "la cultura adhocrática es la cultura dominante en las Mipymes de Durango Capital" y "la ceguera es la incapacidad de aprendizaje que se presenta con mayor frecuencia en las Mipymes de Durango Capital", podemos afirmar que, con base a los resultados obtenidos, la adhocrática es la cultura dominante en el sector industrial estudiado y que la incapacidad de aprendizaje que se presenta con mayor frecuencia es la ceguera.

Es importante comentar que la cultura adhocrática es común para dos tipos de empresas: las pequeñas de 
recién creación y también de empresas consolidadas con una visión de expansión; en el presente estudio, el $55 \%$ de la muestra fue microempresa y el $30 \%$, pequeña, relativamente de recién creación, dos años de funcionamiento las más antiguas.

Vale la pena asentar que en este tipo de empresas, por sus dimensiones, su estructura organizativa es incipiente, generalmente no tienen manuales de organización, ni cuentan con una diferenciación estricta de actividades, razón por la cual el personal termina siendo multifuncional, es decir, todos los empleados hacen de todo; lo anterior solo es una de las características de la cultura adhocrática.

En adición a esto, Cameron y Quinn (2006) destacan que una de las características de la cultura es que cambia de tiempo en tiempo, por lo que resulta de gran interés su afirmación de que las empresas tienden a experimentar patrones de cambio cultural predecible a través de su ciclo de vida, así que no es difícil encontrar en una empresa madura una orientación hacia la cultura jerárquica, que en ocasiones es complementada por un enfoque hacia la cultura de mercado, o, como el hallazgo en los resultados de esta investigación, donde resalta la cultura adhocrática, en las micro y pequeñas empresas.
Para entender esto, plantearemos una metáfora, donde asemejamos la cultura de la empresa con el temperamento en las personas, según planteamientos de algunos autores, que consideran que también las organizaciones lo poseen (Bridges, 2000; Fernández y Hogan, 2003, citados en Calderón y Naranjo, 2007).

En este sentido, siempre se presenta un temperamento dominante, pero difícilmente se ostenta puro, sino que generalmente está influenciado en mayor o menor medida por otros temperamentos, que aderezan la forma de hacer y de reaccionar ante los acontecimientos, de la misma forma una empresa tiene una cultura dominante, que es acompañada en diferentes intensidades de las características de otros tipos de culturas, perfilando con esto, si pudiésemos llamarlo de esta manera, la personalidad propia de la empresa, que además estaría influida por el estilo que el líder le imprima, lo fuerte o no de la misión y sus valores, así como la forma en la que enfrentan los compromisos con el mercado y sus clientes.

Además, no debemos de olvidar que la segunda cultura en intensidad que se presenta es la de clan, con una frecuencia de 65; en el modelo de Cameron y Quinn (2006), las culturas adhocráticas y clan comparten un 
elemento en común, la flexibilidad, pues mientras la primera pone de manifiesto la creatividad de sus miembros, en la segunda, por la escasez de manuales de procedimientos y por no contar con reglamentos, termina el personal reaccionado de manera similar a una ahdhocrática; solo hay una diferencia, en esta última el personal cuenta con una madurez esencial en el desempeño de su trabajo, que no tiene en la segunda.

La tercera cultura que destaca muy de cerca de la segunda es la jerárquica, que habla de empresas con controles fuertes en la toma de decisiones por parte de los dueños, delegando poco o nulo espacio a los empleados, el cual es el caso típico de las micro y pequeñas empresas. Otra característica que tiene esta cultura es estar atenta más al interior que al exterior, estas empresas están más preocupadas por resolver su problemática cotidiana de rotación de personal, de afinar el proceso productivo, de ver o completar el pago de la nómina o de los proveedores, $\mathrm{y}$ las cuestiones hacia el exterior están fuera de su radio de atención.

Por otro lado, la incapacidad que se presenta con mayor frecuencia en las empresas estudiadas es la ceguera, que coincide con el comentario anterior, pues esta barrera impide ver de manera holística a la empresa y, como consecuencia, le imposibilita dar respuestas asertivas a la problemática que se vive, pues quienes la padecen son malos para percibir las amenazas y las oportunidades del entorno (Yeung et al., 2000).

Argyris (1999) afirma que todas las organizaciones generan en mayor o menor medida conocimiento organizacional, sean conscientes de esto o no, y que son las incapacidades las que impiden que el conocimiento se genere o se generalice de mejor manera. Al respecto se destaca que las incapacidades que con mayor frecuencia se presentan son las que impiden la generación del conocimiento, es decir, entre la ceguera, candidez, homogeneidad y acoplamiento estrecho, acumulan el $73 \%$ de las frecuencias y son las barreras que se oponen a que el conocimiento se genere; esto habla de que estas empresas tienen un problema severo para aprender y para trazar estrategias que les permitan mantenerse en el mercado, quizás esta es una de las razones de tanta mortandad empresarial.

Debemos tener en cuenta que la presencia incluso de una sola incapacidad puede ser suficiente para disminuir o bloquear el aprendizaje general en una organización (Gómez-Romero, 2008; Pérez y Cortés, 
2007), y que las incapacidades son como enfermedades silenciosas que invaden a la organización, sin que sus dueños o gerentes se den cuenta de qué es lo que sucede, y en la práctica terminan repercutiendo en la generación o en la generalización del aprendizaje.

Con respecto a la tercera hipótesis de trabajo: existe una relación significativa entre la cultura empresarial y las incapacidades de aprendizaje organizacional en las Mipymes de Durango Capital; al respecto podemos afirmar que existe evidencia estadística de la relación significativa de la cultura con la presencia de las incapacidades de aprendizaje, que concuerda con los criterios de Quinn y Rohrbauch (1983), Yeung et al. (2000) y Gómez-Romero (2008).

De los resultados obtenidos, podemos comentar que en las empresas con cultura clan, las incapacidades que se relacionan con mayor intensidad son la difusión deficiente en primer lugar y la ceguera en segundo lugar. En las empresas con cultura mayormente adhocrática, las barreras más frecuentes son la difusión deficiente, la homogeneidad y el acoplamiento estrecho.

Por su lado, en las empresas con cultura mercado, se observa que son proclives para que se presente con poca intensidad la difusión deficiente y la ceguera; $y$, por último, en la cultura jerárquica, destaca con mucha intensidad la ceguera.

Hasta aquí podemos concluir que se ha observado que, dependiendo del tipo de cultura, será la presencia o no de determinadas incapacidades, $\mathrm{y}$ este es un tema que debe preocupar a los dueños, gerentes y estudiosos del aprendizaje organizacional, pues las barreras impiden que el conocimiento se genere de mejor manera en las empresas.

Las limitaciones de esta investigación están ligadas a la actitud de los directivos, pues al no ser temas supuestamente vitales para la supervivencia de sus empresas, se les presta poca atención, amén que esto dificulta la recopilación de la información, dado que al ignorar la trascendencia de la cultura y de las incapacidades de aprendizaje, no se capacitan ni mejoran y solo se sobreponen al vendaval de la competencia, que cada día amenaza con dejarlos fuera de los mercados de trabajo.

Al difundir los resultados y las conclusiones a las que se han llegado, pretendemos que se puedan discutir con los empresarios del sector, para sensibilizarlos de los peligros organizacionales a los que se enfrentan y hacer un llamado para que se pre- 
paren, sean conscientes de este tema y estén dispuestos a construir organizaciones más eficientes, que den respuestas adecuadas a un entorno, como el que se vive hoy en día, pero, sobre todo, que estén dispuestos a combatir las incapacidades de aprendizaje que afectan a sus empresas. Si logramos esto, entonces estaremos en los albores de presenciar organizaciones más completas y competitivas, capaces de gestar conocimiento organizacional.

Derivado del estudio, quedan algunas interrogantes en el tintero para estudios subsecuentes que se pueden realizar: investigar cómo influye la cultura empresarial en las estrategias competitivas que utilizan como negocio, cómo influye en los estilos de aprendizaje y como estos en la competitividad, en la resistencia al cambio y a la innovación, entre otros temas.

\section{REFERENCIAS}

Alcover de la Hera, C. (2002). El contrato psicológico. El componente implícito de las relaciones laborales.

Alles, M. (octubre, 2001). Selección por competencias. Management Today en español, 26-37.

Argote, L. (2013). Organizational learning $\left(2^{\text {nd }}\right.$ ed.). London: Springer.
Argyris, C. (1991). Teaching smart people how to learn. Harvard Business Review, 69(3).

Argyris, C. (1993). Cómo vencer las barreras organizativas. España: Ed. Díaz de Santos.

Argyris, C. (1999). Sobre el aprendizaje organizacional. México: Ed. Oxford University Press.

Argyris, C., \& Schön, D. A. (1998). Organizationallearning. Madrid: Addison Wesley.

Armenteros Acosta, M., Guerrero Ramos, L., Noyola del Río, F. G., \& Molina, V. (2012). Cultura organizacional y organización que aprende. Un análisis desde la perspectiva de la innovación. $R e$ vista Internacional de Administración y Finanzas, 5(1), 33-51. Audretsch, D., \& Mahmood, T. (1995). New firm survival: new results using a hazard function. Review of Economics \& Statistics, 77(1).

Barraza-Macías, A. (2008). Confiabilidad. Revista INED, (6).

Bohmer, R., \& Edmonson,A. (2001). Organizational learning in health care. Health Forum Journal, 44(2), 72-93.

Bounfour,A.,\&Edvinsson,L.(2005). Intelectual capital for communities. San Francisco: Ed. Elsevier. Bridges, W. (2000). The character of organizations: using Jungian type in organizational development. Pennsylvania: Consulting Psychologists Press. 
Bustillo, C. (2003). Gestión estratégica de personas basadas en competencias. Capital Humano, 164, 14-32.

Calderón, G., \& Naranjo, J. (2007). Perfil cultural de las empresas innovadoras. Un estudio de caso en empresas metalmecánicas. Cuadernos de Administración, 20(39), 161-189.

Cameron, K., \& Quinn, R. (2006). Diagnosing and changing organizational culture. San Francisco: Ed. Jossey-Bass.

Cayeux, A. (1964). ¿Qué es el evolucionismo? México: Ed. Planeta.

Choo, W. (1999). La organización inteligente: el empleo de la información para dar significado, crear conocimiento y tomar decisiones. México: Ed. Oxford University Press.

Daft, R. (1998). Teoría y diseño organizacional. México: Ed. Soluciones Empresariales.

Daft, R. L. (2000). Organization theory and design. South, USA.

Dallago, B. (2003). The importance of small and medium enterprises. U.N. Chronicle, United Nations Publications, (4), 213-226.

Deal, T., \& Kennedy, A. (2000). Corporate cultures. New York: Perseus Books Publishing.

Díaz-Nieto, E. S. (2008). Modelo de cultura organizacional para promover la ventaja competitiva empresarial. El caso papeles ponderosa (San Juan del Río, Queré- taro, México). Revista Gallega de Economía, 17(1), 257-264.

Furtado, C. (2003). La transición de la economía mexicana. III Conferencia Internacional de la Red de Estudios sobre el Desarrollo, México.

Geranmayeh, A. (1992). Organizational learning through interactive planning: design of learning systems for ideal seeking organizations (Tesis doctoral, Universidad de Pennsylvania, USA).

Gómez, J. G., Villareal Solís, M. F., \& Villarreal Solís, M. D. (2014). Las incapacidades de aprendizaje organizacional y su relación con los estilos de aprendizaje organizacional en la Mipyme de la ciudad de Durango, México. Revista Contaduría y Administración.

Gómez-Romero, J. G. (2008). La generación de conocimiento organizacional en la micro, pequeña y mediana empresas (Mipymes) de Durango. Durango: Editorial UJED.

Gooderham, P. (2007). Enhancing knowledge transfer in corporations: adynamic capabilities drive model. Knowledge Management Research \& Practice, 5, 34-43.

Guzzeta, M. (2008). Modelo teórico para la determinación del impacto tecnológico sobre la cultura organizacional de las Pymes manufactureras de Barquisimeto. Gestión y Gerencia, 1(1). 
Hair, J. F., Anderson, R. E., Tatham, R. L., \& Black, W.C.(2005). Data analysis with readings. New Jersey: Prentice Hall International. Hernández, M., Mendoza, J., \& González, L. (2008). Construcción y validez del instrumento de cultura organizacional y competitividad (ICOC). En J. Pintado \& G. Payne (Coords.). Estableciendo puentes en una economía global. Vol. 2. Recuperado de http://dialnet.unirioja.es/servlet/ articulo? codigo $=2734274$

Hernández,R.,Fernández,C.,\&Baptista, P. (2006). Metodología de la investigación. McGraw-Hill.

Hernández, R., Méndez, S., \& Contreras, R. (enero-marzo, 2014). Construcción de un instrumento para medir el clima organizacional en función del modelo de los valores en competencia. Contaduría y Administración, 59(1), 229-257.

Hernández-Romo, M. (2004). La cultura empresarial en México. México: Ed. Universidad Autónoma de Aguascalientes.

Huntington, E. (2001). La tercera vía. México: Ed. Diana.

INEGI. (2006). Perspectiva económica de Durango. México: Publicaciones del INEGI.

INEGI. (2014). Directorio Estadístico Nacional de Unidades Económicas (DENUE). México: $\mathrm{Pu}-$ blicaciones del INEGI.
INEGI. (2014). Estudio de la micro, pequeña y mediana empresa en Durango. México: Publicaciones del INEGI.

INEGI. (2015). Directorio del Sistema de Información Empresarial Mexicano (SIEM) del INEGI.

Lind, D. A., Marchal, W. G., \& Wathen, S. A. (2005). Statistical techniques in business \& economics. New York: McGraw-Hill.

Lounamaa, P., \& March, J. G. (1987). Adaptative coordination of learning team. Management Science, 33(1), 107-123.

Palomo Garrido, A. (2011). La globalización y su renombre. Nómadas, (32).

Pérez Zapata, J., \& Cortés Ramírez, J. A. (2007). Barreras para el aprendizaje organizacional. Estudio de casos. Pensamiento \& Gestión, (22).

Pfeffer, J. (2000). Nuevos rumbos en la teoría de la organización. Problemas y posibilidades. México: Oxford University Press.

Prater, E., \& Ghosh, S. (2005). Small and medium-sized enterprises in Europe. Journal of Small Business Management, 43(2), 155. Probst, G., \& Büchel, B. (1995). La practique de l'enterprise apprenante. Francia: Les editions d'organisation.

Probst, G., Raub, S., \& Romhardt, K. (2001). Administre el conocimiento. "Los pilares del éxito”. México: Ed. Prentice Hall. 
Quinn, J. B., Anderson, P., \& Finkelstein, S. (1998). Managing professional intellect: making the most of the best. In The strategic management of intellectual capital (pp. 87-100).

Quinn, R. E., \& McGrath, M. R. (1985). The transformation of organizational cultures: a competing values perspective. In Organizational culture (pp. 315-334).

Quinn, R., \& Rohrbauch, Y. (1983). A spatial model of effectiveness criteria: toward a competing values approach to organizacional analisis. Management Sciences, 29(3).
Rodríguez, M., \& Ramírez P. (2004). Psicología del mexicano en el trabajo. México: Ed. McGraw-Hill. Senge, P. (1993). La quinta disciplina. México: Ed. Granica.

Thevenet, M. (1992). Auditoría de la cultura empresarial. Barcelona: Ed. Díaz de Santos.

Watkins, K. E., \& Marsick, V. J. (1993). Sculpting the learning organization. USA: Ed. Jossey-Bass. Yeung, A., Ulrich, D., Nason, S., \& Von Glinow, M.A.(2000). Las capacidades del aprendizaje en la organización: cómo aprender a generar ideas con impacto. México: Ed. Oxford University Press. 